\title{
Implantation Chain Dosage Form
}

National Cancer Institute

\section{Source}

National Cancer Institute. Implantation Chain Dosage Form. NCI Thesaurus. Code C149563.

Solid sterile preparation consisting of small spheres mounted on a non-degradable thread to form a chain that allows withdrawal of the remainder of the chain after a certain period of action. Each implantation chain is presented in a sterile container. The implantation chain is intended for release over an extended period of time in order to obtain local or systemic effect. 